\title{
Prepubertal ovariectomy alters dorsomedial striatum indirect pathway neuron excitability and explore/exploit balance in female mice
}

3

5
Kristen Delevich ${ }^{1,2^{*}}$, Christopher D. Hall ${ }^{1}$, and Linda Wilbrecht ${ }^{1,2^{*}}$

1 Department of Psychology, University of California, Berkeley, CA 94720, USA

2 Helen Wills Neuroscience Institute, University of California, Berkeley, CA 94720, USA

*co-corresponding author

Linda Wilbrecht, $\mathrm{PhD}$

Department of Psychology

University of California, Berkeley

16 Barker Hall

Berkeley, CA 94720-1650

Tel: +1.510 .600 .3560$

wilbrecht@berkeley.edu

Kristen Delevich, $\mathrm{PhD}$

Department of Integrative Physiology and Neuroscience College of Veterinary Medicine

Washington State University

P.O. Box 647620 Pullman, WA 99164

Tel: +1.509-335-4829

kristen.delevich@wsu.edu 


\section{Abstract:}

Decision-making circuits are modulated across life stages (e.g. juvenile, adolescent, or adult) — as well as on the shorter timescale of reproductive cycles in females - to meet changing environmental and physiological demands. Ovarian hormonal modulation of relevant neural circuits is a potential mechanism by which behavioral flexibility is regulated in females. Here we examined the influence of prepubertal ovariectomy (pOVX) versus sham surgery on performance in an odor-based multiple choice reversal task. We observed that pOVX females made different types of errors during reversal learning compared to sham surgery controls. Using reinforcement learning models fit to trial-by-trial behavior, we found that pOVX females exhibited lower inverse temperature parameter ( $\beta$ ) compared to sham females. These findings suggest that OVX females solve the reversal task using a more exploratory choice policy, whereas sham females use a more exploitative policy prioritizing estimated high value options. To seek a neural correlate of this behavioral difference, we performed whole-cell patch clamp recordings within the dorsomedial striatum (DMS), a region implicated in regulating action selection and explore/exploit choice policy. We found that the intrinsic excitability of dopamine receptor type 2 (D2R) expressing indirect pathway spiny projection neurons (iSPNs) was significantly higher in pOVX females compared to both unmanipulated and sham surgery females. Finally, to test whether mimicking this increase in iSPN excitability could recapitulate the pattern of reversal task behavior observed in pOVX females, we chemogenetically activated DMS D2R(+) neurons within intact female mice. We found that chemogenetic activation increased exploratory choice during reversal, similar to the pattern we observed in pOVX females. Together, these data suggest that pubertal status may influence explore/exploit balance in females via the modulation of iSPN intrinsic excitability within the DMS. 


\section{Introduction:}

As animals interact with their environment in pursuit of rewards in the form of food, water, mates etc., they learn from trial and error to guide their future choices. This process involves learning from positive and negative feedback and also, importantly, deciding how learned information should influence choice, referred to as choice policy. Reinforcement learning (RL) models (Sutton and Barto, 1998) have provided a useful framework for understanding and quantifying aspects of trial-and-error learning, including choice policy. A classic RL problem that hinges on choice policy is the explore/exploit tradeoff. If an animal (or any agent for that matter) adopts an exploit policy, it will consistently select the highest estimated value option, but may miss out on better alternative options. On the other hand, if an animal favors a more exploratory choice policy, characterized by less value-dependent, more stochastic choice behavior, it may discover new and better options more readily (Sutton and Barto, 1998; Daw et al., 2006). Importantly, the optimal balance of exploration and exploitation may depend on the statistics of the environment and/or the needs of the animal as defined by its particular physiological or developmental state (Cohen et al., 2007; Frank et al., 2009; Humphreys et al., 2015; Addicott et al., 2017; Lenow et al., 2017; Gopnik, 2020). In humans, choice behavior generally becomes less exploratory and more exploitative during the transition from childhood to adulthood (Nussenbaum and Hartley, 2019; Gopnik, 2020; Xia et al., 2020; Eckstein et al., 2021). Natural fluctuations in ovarian hormones across the estrous cycle or exogenous estradiol administration have been shown to regulate aspects of value-based decision making in female rats (Uban et al., 2012; Orsini et al., 2021), including explore/exploit balance (Verharen et al., 2019b). These data suggest that the rise in ovarian hormones at puberty could contribute to the developmental shift in choice policy during

4 adolescence in females. In previous work, we observed that pOVX altered performance in a 
95 multiple choice reversal task in adult C57/B16 mice (Delevich et al., 2020a). Compared to intact

96 females, pOVX females showed lower ratios of perseverative to regressive errors during reversal

97 learning, but the potential underlying biological processes that contributed to this behavioral effect

98 remained unclear.

The DMS is implicated in the regulation of goal-directed action selection (Tai et al., 2012;

100 Nonomura et al., 2018; Matamales et al., 2020; Peak et al., 2020) and choice policy (Collins and

101 Frank, 2014), and recent work suggests that enhancing the activity of D2R(+) SPNs in the dorsal

102 striatum biases choice behavior to be more exploratory (Lee et al., 2015; Delevich et al., 2020b)

103 but see (Verharen et al., 2019a). While nuclear estrogen receptors are notably absent from the

104 dorsal striatum in adulthood (Krentzel et al., 2021), extranuclear estrogen receptors (ER $\alpha$, ER $\beta$,

105 and GPER1) localize to SPNs, glia, and the presynaptic terminals of striatal GABAergic and

106 cholinergic interneurons of adult female rats (Almey et al., 2012). At the neuronal level, estrous

107 cycle has been shown to regulate the intrinsic excitability of SPNs located within the rodent

108 striatum (Proano et al., 2018; Alonso-Caraballo and Ferrario, 2019). Studies examining the

109 influence of estrous cycle on SPN physiology have been primarily performed in rats, where SPN

110 cell types were not distinguished, but see (Tansey et al., 1983). Taken together, these findings raise

111 the question of whether pubertal status influences choice strategies employed by females by

112 modulating striatal SPN physiology. Here we focused on D2R(+) SPNs of the indirect pathway

113 (iSPNs) within the DMS, whose activity we hypothesized regulates explore/exploit balance in

114 decision making based on theoretical predictions (Collins and Frank, 2014; Dunovan and

115 Verstynen, 2016) plus genetic (Beeler et al., 2010; Kwak et al., 2014) and pharmacological (Lee

116 et al., 2015; McCoy et al., 2019) evidence. 
In the current study, after analyzing raw behavioral data, we applied reinforcement learning

118 modeling to examine how pOVX influenced learning and choice policy processes underlying

119 performance in the odor-based reversal learning task. We next examined the influence of pOVX

120 on the intrinsic excitability of genetically identified D2R(+) SPNs within the DMS of adult female

121 mice. Finally, we chemogenetically activated D2R $(+)$ neurons within the DMS of female mice

122 during reversal learning and applied our RL model to determine whether this manipulation

123 recapitulated the reversal learning strategy employed by pOVX females. We found that compared

124 to intact adult females, pOVX females exhibited a more exploratory choice strategy during reversal

125 learning as evidenced by a lower explore/exploit inverse temperature parameter, $\beta$. In addition,

$126 \mathrm{D} 2 \mathrm{R}(+)$ SPN intrinsic excitability was increased in pOVX females compared to sham females.

127 Finally, chemogenetic activation of D2R(+) SPNs within the DMS promoted a more exploratory

128 choice strategy during reversal learning in intact female mice, resembling pOVX female behavior.

129 Together, these data suggest that pubertal status influences the choice strategy female mice employ

130 via the modulation of D2R(+) SPN activity.

131 Materials \& Methods:

132 Animals:

Female C57BL/6NCR (Charles River), Drd2-eGFP BAC (GENSAT), and D2-Cre ER43

134 (MMRC) mice were bred in-house. Drd2-eGFP BAC and D2-Cre ER43 mice were bred onto the

135 C57BL/6NCR background for at least 5 generations. All mice were weaned on postnatal day (P)21

136 and housed in groups of 2-3 same-sex siblings on a 12:12 hr reversed light:dark cycle (lights on

137 at $2200 \mathrm{~h}$ ). All behavioral tests were conducted during the dark phase. For all experiments, mice

138 were randomly assigned to experimental groups and sample sizes were based on previously

139 conducted experiments (e.g. Delevich et al. 2020a,b). Each behavioral experiment was conducted 
140 once, and no animal was tested on multiple occasions. All procedures were approved by the

141 Animal Care and Use Committee of the University of California, Berkeley and conformed to

142 principles outlined by the NIH Guide for the Care and Use of Laboratory Animals.

143 Prepubertal Ovariectomy:

144 Prepubertal ovariectomy was performed as previously described (Delevich et al., 2020a).

145 To eliminate ovarian hormone exposure during and after puberty, ovariectomies were performed

146 before puberty onset at P25. Prior to ovariectomy (OVX) surgery, all female mice were visually

147 inspected to confirm that vaginal opening had not occurred. Prior to surgery, mice were injected

148 with $0.05 \mathrm{mg} / \mathrm{kg}$ buprenorphine and $10 \mathrm{mg} / \mathrm{kg}$ meloxicam subcutaneously and were anesthetized

149 with 1-2\% isoflurane during surgery. The incision area was shaved and scrubbed with ethanol and

150 betadine. Ophthalmic ointment was placed over the eyes to prevent drying. A $1 \mathrm{~cm}$ incision was

151 made with a scalpel in the lower abdomen across the midline to access the abdominal cavity. The

152 ovaries were clamped off from the uterine horn, with locking forceps and ligated with sterile

153 sutures. After ligation, the ovaries were excised with a scalpel. The muscle and skin layers were

154 sutured, and wound clips were placed over the incision for 7-10 days to allow the incision to heal.

155 An additional injection of $10 \mathrm{mg} / \mathrm{kg}$ meloxicam was given 24 and $48 \mathrm{~h}$ after surgery. Sham control

156 surgeries were performed in which fat pads were visualized but the ovaries were not clamped,

157 ligated, or excised. Female littermates were randomly assigned to sham or pOVX groups. Mice

158 were allowed to recover on a heating pad until ambulatory and were post-surgically monitored for

$1597-10$ days to check for normal weight gain and signs of discomfort/distress. Mice were co-housed

160 with 1-2 siblings who received the same surgical treatment. To confirm the success of prepubertal

161 ovariectomies, necropsy was performed on a subset of adult sham and ovariectomized mice to 
162 confirm that the uteri of pOVX mice were underdeveloped compared to age-matched sham females

163 (data not shown).

1644 choice odor-based reversal task:

165 Sham or pOVX mice were tested in an odor-based reversal task that has previously been

166 described in detail (Johnson and Wilbrecht, 2011; Johnson et al., 2016) as young adults (P60-P70).

167 The task is designed such that only the odor cue is predictive of reward, while spatial and 168 egocentric information is irrelevant. Briefly, mice were food restricted to $\sim 85 \%$ body weight by 169 the Discrimination phase. Mice were habituated to the testing arena on day 1, they were taught to 170 dig for a honey nut cheerio reward in a pot filled with unscented wood shavings on day 2 , 171 underwent a 4 choice odor Discrimination on day 3, and finally, were tested on Recall of the 172 previously learned odor-reward association, which was immediately followed by a Reversal phase 173 on day 4. During the Discrimination phase of the task, mice learned to discriminate among four 174 pots with different scented wood shavings (anise, clove, litsea, and thyme). All four pots were 175 sham-baited with cheerio (under wire mesh at bottom) but only one pot was rewarded (anise). The 176 pots of scented shavings were placed in each corner of an acrylic arena (12", 12", 9") which was

177 divided into four quadrants. Mice were placed in a cylinder in the center of the arena, and a trial 178 started when the cylinder was lifted. Mice were then free to explore the arena and indicate their 179 choice by making a bi-manual dig in one of the four pots of wood shavings. The cylinder was 180 lowered as soon as a choice was made. If the choice was incorrect, the trial was terminated and the 181 mouse was gently encouraged back into the start cylinder. Trials in which no choice was made 182 within 3 minutes were considered omissions. If mice omitted for two consecutive trials, they 183 received a reminder: a baited pot of unscented wood shavings was placed in the center cylinder 184 and mice dug for the "free" reward. Mice were disqualified if they committed four pairs of 
185 omissions. The location of the four odor scented pots was shuffled on each trial, and criterion was

186 met when the mouse completed 8 out of 10 consecutive trials correctly. 24 hours after completing

187 Discrimination, mice were tested for Recall of the initial odor Discrimination to criterion, after

188 which, mice immediately proceeded to the Reversal phase in which the previously rewarded odor

189 (anise) was no longer rewarded, and a previously unrewarded odor (clove) was now rewarded.

190 During the Reversal phase, Odor 4 (thyme) was replaced by a novel odor (eucalyptus) that was

191 unrewarded. Again, mice were run until they reached a criterion of 8 out of 10 consecutive correct

192 trials.

1934 choice odor-based reversal task analysis:

194 To compare reversal task performance across groups, trials to criterion and errors (incorrect

195 choices) were compared for each phase of the task (Discrimination, Recall, and Reversal).

196 Omission trials did not count towards trials to criterion. In addition, for the Reversal phase we

197 separated errors in which mice chose the odor that was rewarded during Discrimination (Odor 1)

198 into two types: 1) perseverative errors occurred when Odor 1 was chosen prior to the first correct

199 trial and 2) regressive errors occurred when Odor 1 was chosen after the first correct trial during

200 the Reversal phase. To compare the relative proportion of these error types within mice, we

201 calculated Reversal error bias as (perseverative - regressive errors)/(perseverative + regressive

202 errors). Therefore, a value $>1$ indicates a bias for perseverative errors whereas a value $<1$ indicates

203 a bias for regressive errors. Finally, we examined how quickly mice accumulated rewards after the

204 first correct trial during the Reversal phase by aligning trial histories to the first correct trial and 205 summing rewarded trials across the subsequent 8 trials. Data were fit by linear regression for each

206 group and the slope of the lines compared to determine whether groups significantly differed in

207 their rate of reward accumulation. Behavioral data from 14 of the 16 pOVX females and 10 of the 
20815 sham females presented here were included in a previously published study examining sex

209 differences of prepubertal gonadectomy on approach-avoidance behaviors, but latent decision

210 variables were not examined (Delevich et al., 2020a).

\section{Reinforcement learning modeling of 4 choice odor-based reversal task}

We modeled Discrimination and Reversal phase behavior using a reinforcement learning

213 model driven by an iterative error-based rule (Rescorla and Wagner, 1972; Sutton and Barto,

214 1998). The model uses a prediction error $(\delta)$ to update the value $(V)$ of each odor stimulus, where

$215 \delta$ is the difference between the experienced feedback $(\lambda)$ and the current expected value $(r=100$

216 for rewarded, $\mathrm{r}=0$ for unrewarded) scaled by a learning rate parameter $(\alpha)$, with $0<\alpha<1$ :

$$
V(t+1)_{\text {choice }}=V(t)_{\text {choice }}+\alpha \times \delta(t)
$$$$
\delta(t)=r(t)-V(t)_{\text {choice }}
$$

$$
r(t)\left\{\begin{array}{c}
100 \text { if rewarded choice } \\
\text { else } 0
\end{array}\right.
$$

Because mice exhibit innate preferences for odors, we set initial odor values to fixed

223 during the first 4 trials of Discrimination $\times 100$ (see Johnson et al. 2016). These initial odor values

224 were calculated separately for mice included in Figure 1 and Figure 4 (see data source files or

225 analysis code for more details). To model trial-by-trial choice probabilities, the stimulus values

226 were transformed using a softmax function to compute choice probabilities based on estimated

227 odor values, $V(O)_{i}$. The inverse temperature parameter $(\beta)$, which we refer to in the text as the

228 explore/exploit parameter, determined the stochasticity of the choices:

$$
\sigma(V(O))_{i}=\frac{e^{\beta V(O)_{i}}}{\sum_{i=1}^{n} e^{\beta V(O)_{i}}}
$$


For RL modeling, trial histories from Discrimination and Recall phases were concatenated

231 to create one Discrimination phase trial history. We compared the alternative models using AIC

232 (Watanabe, 2010) and found that the best fit model included phase-specific (non-zero) $\alpha$ and $\beta$

233 parameters; all RL model comparisons for pOVX and sham females are presented in Table S1 as

234 well as source data files and analysis code. To assess model performance, trial-by-trial behavioral

235 data was recovered using the best fit parameters for each animal, and average recovered choices

236 to criterion for Discrimination and Reversal phases (100 simulations/animal) were plotted against

237 the actual choices to criterion for each animal.

\section{Stereotaxic Virus Injection}

Female D2-Cre mice (6-8 weeks) were deeply anesthetized with 5\% isoflurane (vol/vol) in

240 oxygen and placed into a stereotactic frame (Kopf Instruments; Tujunga, CA) upon a heating pad.

241 Anesthesia was maintained at 1-2\% isoflurane during surgery. An incision was made along the

242 midline of the scalp and small burr holes were drilled over each injection site. Virus was delivered

243 via microinjection using a Nanoject II injector (Drummond Scientific Company; Broomall, PA).

244 Injection coordinates for DMS were (in mm from bregma): 0.90 anterior, +/-1.4 lateral, and -3.0

245 from surface of the brain. Adeno-associated viruses (AAVs) were produced by Addgene viral

246 service and had titers of $>10^{12}$ genome copies per $\mathrm{mL}$. For chemogenetic manipulations, mice were 247 bilaterally injected with $0.5 \mathrm{uL}$ of rAAV8-hsyn-DIO-mCherry (N=9) rAAV8-hsyn-DIO-hM3Dq-

248 mCherry $(\mathrm{N}=6)$, or rAAV8-hsyn-DIO-hM4Di-mCherry $(\mathrm{N}=5)$. Mice were given subcutaneous 249 injections of meloxicam $(10 \mathrm{mg} / \mathrm{kg})$ during surgery and 24 and 48 hours after surgery. Mice were 250 group-housed before and after surgery and 4-6 weeks were allowed for viral expression before 251 behavioral training or electrophysiology experiments. 
254 Supply Program (NIMH C-929). CNO was made fresh each day and dissolved in DMSO $(0.5 \%$

255 final concentration) and diluted to $0.1 \mathrm{mg} / \mathrm{mL}$ in $0.9 \%$ saline USP.

\section{Electrophysiology}

Mice were deeply anesthetized with an overdose of ketamine/xylazine solution and

258 perfused transcardially with ice-cold cutting solution containing (in $\mathrm{mM}$ ): 110 choline-Cl, $2.5 \mathrm{KCl}$,

$2597 \mathrm{MgCl} 2,0.5 \mathrm{CaCl} 2,25 \mathrm{NaHCO} 3,11.6 \mathrm{Na}$-ascorbate, 3 Na-pyruvate, $1.25 \mathrm{NaH} 2 \mathrm{PO}$, and $25 \mathrm{D}-$

260 glucose, and bubbled in 95\% O2/5\% CO2. $300 \mu \mathrm{m}$ thick coronal sections were cut in ice-cold

261 cutting solution before being transferred to ACSF containing (in $\mathrm{mM}$ ): $120 \mathrm{NaCl}, 2.5 \mathrm{KCl}, 1.3$

$262 \mathrm{MgCl} 2,2.5 \mathrm{CaCl} 2,26.2 \mathrm{NaHCO} 3,1 \mathrm{NaH} 2 \mathrm{PO} 4$ and 11 Glucose. Slices were bubbled with 95\%

$263 \mathrm{O} 2 / 5 \% \mathrm{CO} 2$ in a $37^{\circ} \mathrm{C}$ bath for $30 \mathrm{~min}$, and allowed to recover for $30 \mathrm{~min}$ at room temperature

264 before recording. All recordings were made using a Multiclamp 700B amplifier and were not

265 corrected for liquid junction potential. The bath was heated to $32^{\circ} \mathrm{C}$ for all recordings. Data were

266 digitized at $20 \mathrm{kHz}$ and filtered at 1 or $3 \mathrm{kHz}$ using a Digidata 1440 A system with pClamp 10.2

267 software (Molecular Devices, Sunnyvale, CA, USA). Only cells with access resistance of $<25 \mathrm{M} \Omega$

268 were retained for analysis. Cells were discarded if parameters changed more than 20\%. Data were

269 analyzed using pClamp or R (RStudio 0.99.879; R Foundation for Statistical Computing, Vienna,

270 AT).

271 Whole-cell current clamp recordings were performed using a potassium gluconate-based

272 intracellular solution (in $\mathrm{mM}$ ): $140 \mathrm{~K}$ Gluconate, $5 \mathrm{KCl}, 10 \mathrm{HEPES}, 0.2 \mathrm{EGTA}, 2 \mathrm{MgCl} 2,4$

273 MgATP, 0.3 Na2GTP, and 10 Na2-Phosphocreatine. Alexa Fluor $594(40 \mu \mathrm{M})$ was added to the

274 internal solution to enable morphological confirmation of SPN identify following recording. In

275 order to block NMDA and AMPA-mediated currents, $5 \mu \mathrm{M}$ AP5 and $25 \mu \mathrm{M}$ NBQX were added 
276 to the ACSF, respectively for intrinsic excitability data in Figure 2. For all recordings, cells were

277 allowed to stabilize for 2 min after break in and prior to any current injection. For current clamp

278 recordings to test the effect of $\mathrm{CNO}$ in Gq-DREADD- expressing vs. mCherry-expressing D2R(+)

279 neurons, baseline input-output curves were collected before 5 minute wash-on of $10 \mu \mathrm{M} \mathrm{CNO}$.

280 Histology

Mice were transcardially perfused with PBS followed by 4\% PFA in PBS. Following 24h

282 postfixation, coronal brain slices $(75 \mu \mathrm{m})$ were sectioned using a vibratome (VT100S Leica

283 Biosystems; Buffalo Grove, IL). To confirm viral targeting, we performed a standard

284 immunohistochemical procedure using a primary antibody against red fluorescence protein (RFP)

285 (rabbit, Rockland 600-401-379; 1:1000) to enhance the mCherry signal expressed in mice

286 transduced with rAAV8-hSyn-DIO-DREADD-mCherry or rAAV8-hSyn-DIO-mCherry. Sections

287 were counterstained with DAPI (Life Technologies; Carlsbad, CA). Images were acquired with a

288 Zeiss Axio Scan.Z1 epifluorescence microscope (Molecular Imaging Center, UC Berkeley) at 10x

289 magnification and viewed using FIJI (ImageJ). Anatomical regions were identified according to

290 the Mouse Brain in Stereotaxic Coordinates by Franklin and Paxinos and the Allen Institute Mouse

291 Brain Atlas.

292 Statistics and Data Analysis

293 For comparisons between 2 groups, a t-test was used when data were normally distributed, and

294 Welch's correction was applied when variance was unequal. The D'Agostino \& Pearson test was

295 used to test for normality. For experiments in which 3 groups were compared, a one-way ANOVA

296 or Kruskal Wallis test when not normally distributed was performed, followed by two-tailed

297 uncorrected Fisher's LSD or Dunn's test, respectively, for pairwise comparisons. Two-way

298 ANOVA was performed when two independent variables were examined (e.g. treatment and error 
type), followed by uncorrected Fisher's LSD (two-tailed) for pairwise comparisons. Post-hoc comparisons were not corrected, due to the limited number of planned comparisons. Throughout

301 the paper, $\mathrm{p}=0.05$ was used as the criterion for a significant statistical difference unless noted

302 otherwise. Data are expressed as mean \pm SEM unless noted otherwise.

\section{Data availability}

304 All data generated or analyzed during this study are included in the manuscript and supporting

305 files. Source data files have been provided for all experiments reported in this manuscript in an

306 online repository at https://doi.org/10.6084/m9.figshare.14783628.v1. Analysis code is available

307 at https://github.com/kdelevich/4choice RLmodeling.

308 Results:

309 Prepubertal ovariectomy affects reversal learning by promoting exploratory choice policy

310 We performed sham surgery or pOVX on female C57/B16 mice at postnatal day 25 (P25),

311 prior to puberty onset, and trained them in an odor-based reversal task between P60-70 (Fig. 1A).

312 The odor-based reversal task consisted of two main phases: 1) a Discrimination phase during

313 which mice learned through trial and error that one of four scented pots of wood shavings contained

314 a buried food reward and 2) a Reversal phase in which the odor-reward contingency was reversed

315 (Fig. 1B). Sham females were not staged for estrous cycle, and both groups performed similarly

316 in the Recall phase (Supplementary Fig. 1). When comparing Discrimination and Reversal, there

317 was a significant effect of task phase but not treatment on trials to reach criterion [task phase:

$318 \mathrm{~F}(1,29)=6.31, \mathrm{p}=0.018$; treatment: $\mathrm{F}(1,29)=0.11, \mathrm{p}=0.74$; task phase $\times$ treatment: $\mathrm{F}(1,29)=0.05$, $319 \mathrm{p}=0.83]$ (Fig. 1C).

320 Next, we more closely examined the types of errors that mice made during Reversal. Error 321 types included those made to the previously rewarded odor, which we divided into 2 subtypes: 
perseverative (errors made before the first correct trial) and regressive (errors made after first correct trial). Perseverative errors reflect a tendency to stick to a previously learned rule, whereas

324 regressive errors reflect a failure to acquire or maintain the new rule. There was a significant

325 interaction between error type and treatment group $[F(5,145)=2.79, p=0.02]$ (Fig. 1D). Post hoc analyses revealed that pOVX females made significantly more regressive errors compared to sham

327 females ( $p=0.03$ uncorrected Fisher's LSD). We next examined the pattern of perseverative and regressive errors made by individual mice. Sham females exhibited a significantly higher ratio of perseverative to regressive errors (Reversal error bias) compared to pOVX females (sham vs. pOVX females: $\mathrm{t}(29)=2.12, \mathrm{p}=0.04$ ) (Figure 1E). Finally, we observed that sham females accumulated rewards at a significantly higher rate after the first rewarded trial compared to pOVX females during Reversal but not Discrimination (Figure 1F). These data suggest that sham females and pOVX females reach criterion in the reversal task using different trial-by-trial strategies.

We next turned to computational modeling to assess if differences observed in the Reversal

336 difference in choice policy, or a combination of both. To do so, we fit trial-by-trial behavioral data

337 with RL models and used the maximum log likelihood to determine the parameters that best fit 338 each animal's behavior. The best fit model included phase-specific parameters for the learning rate $339 \alpha$ and the explore/exploit inverse temperature parameter $\beta$ (Fig. 1G) (see Supplementary Table 1 340 for alternate model comparison). We found that there was a significant interaction between task 341 phase and treatment for the explore/exploit parameter $\beta$ [task phase $\times$ treatment: $F(1,29)=7.101$, $342 \mathrm{p}=0.013]$. In sham and pOVX female mice, the explore/exploit parameter was significantly higher 343 during the Reversal phase compared to Discrimination phase (sham Reversal vs. Discrimination: $344 \mathrm{p}<0.0001 ; \mathrm{pOVX}$ Reversal vs. Discrimination $\mathrm{p}=0.011$ uncorrected Fisher's LSD) and Reversal 
345 phase explore/exploit parameter was significantly lower in pOVX vs. sham females (pOVX vs.

346 sham: $\mathrm{p}=0.014$ uncorrected Fisher's LSD) consistent with pOVX females employing a more

347 exploratory choice policy compared to sham females (Fig. 1H).

A

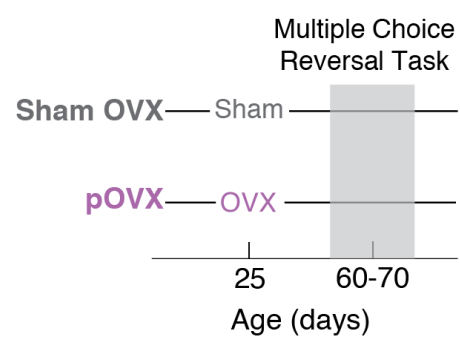

B

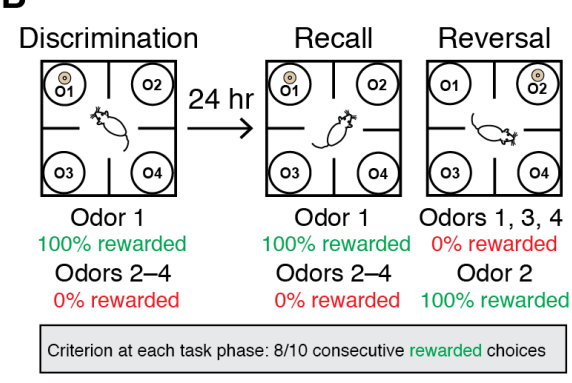

D
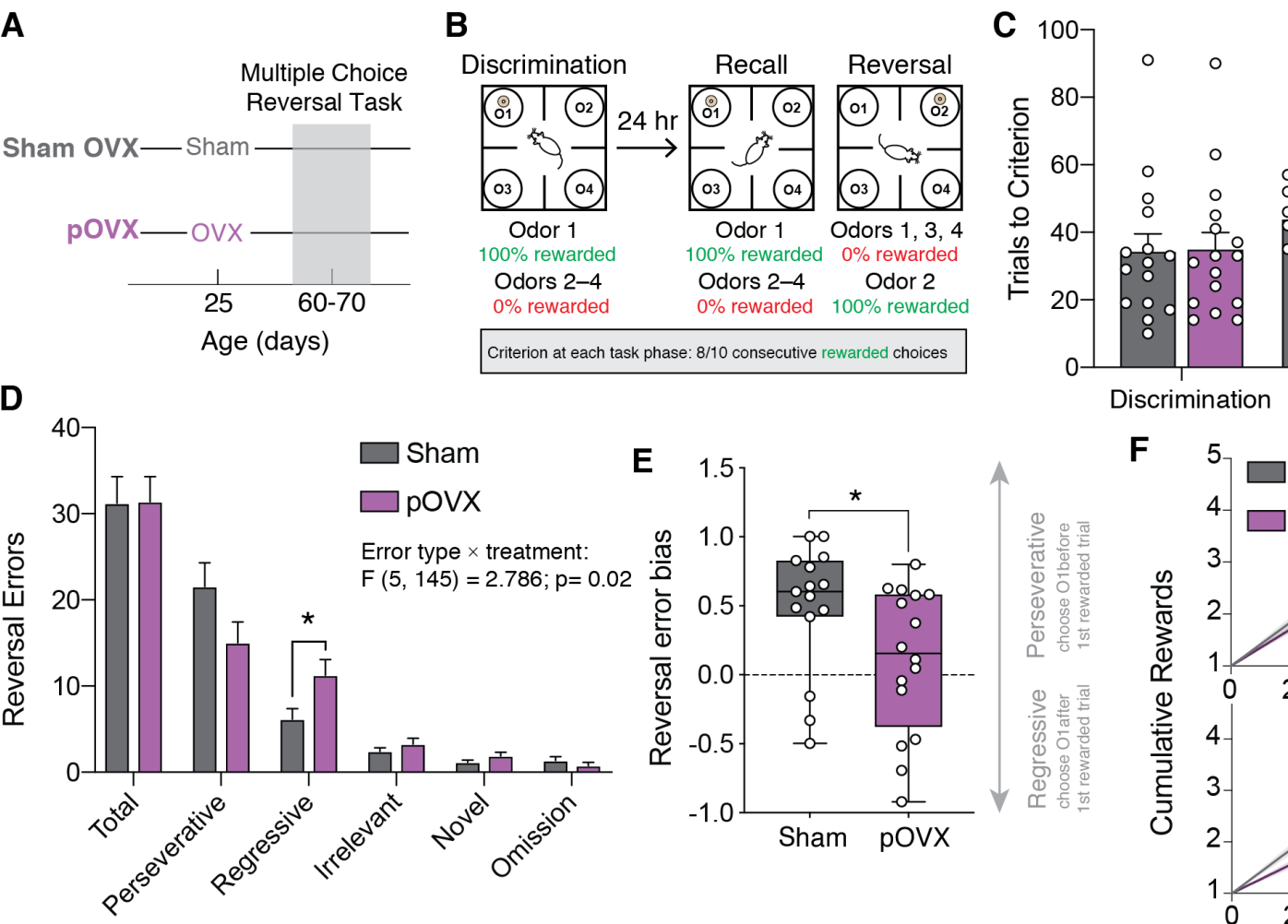

Sham

Discrimination Reversal

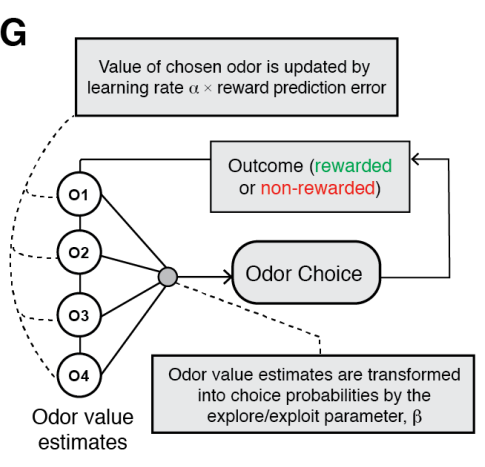

348
H<smiles>[H]</smiles>

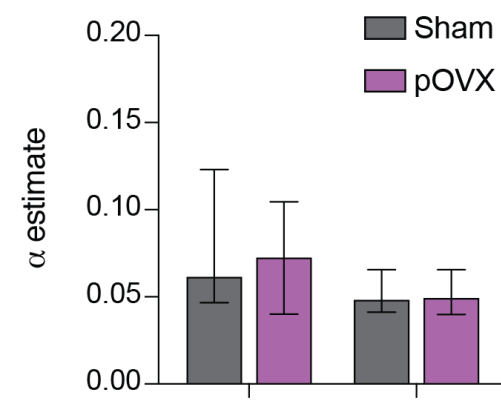

Task phase Discrimination Reversal

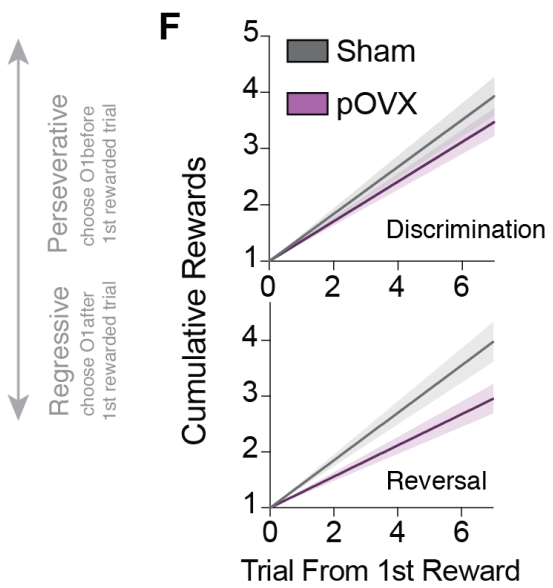

Trial From 1st Reward

I Explore/Exploit parameter

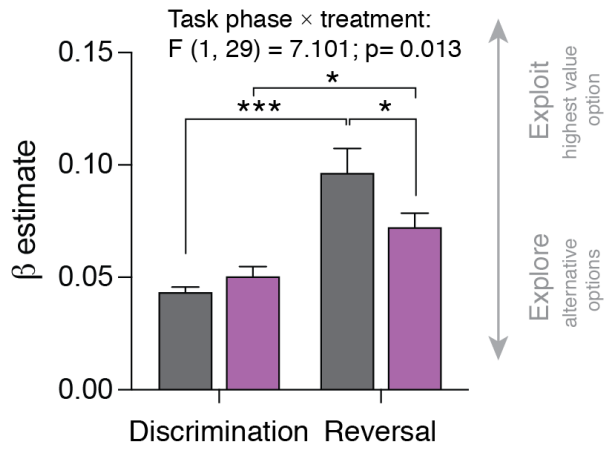

Figure 1. Prepubertal OVX is associated with more exploratory reversal learning strategy in

female mice. (A) Female C57/B16 mice underwent OVX or sham surgery at P25 and were trained in the multiple choice reversal task in adulthood (P60-70). (B) Mice were trained to a criterion of 
8/10 correct consecutive choices to Odor 1 during Discrimination. 24 hours later they were tested

353 for Recall of the previous day's rule before immediately advancing to a Reversal phase during

354 which Odor 2, rather than Odor 1, was rewarded. Reversal criterion was reached when mice made

$3558 / 10$ correct consecutive choices to Odor 2 . (C) There was a main effect of task phase on trials to

356 criterion but no effect of treatment (Two-way RM ANOVA main effect of task phase $\mathrm{F}(1,29)=$

$3576.30, \mathrm{p}<0.05)$. (D) There was a significant effect of treatment and error type on the number of

358 reversal errors (Two-way RM ANOVA treatment $\times$ error type interaction: $\mathrm{F}(5,145)=2.79$,

$359 \mathrm{p}<0.05)$. pOVX females made significantly more regressive errors compared to sham females

360 (11.25 \pm 1.8 vs. $6.13 \pm 1.2, \mathrm{p}<0.05$, uncorrected Fisher's LSD). (E) pOVX females had a

361 significantly lower Reversal error bias (perseverative - regressive errors)/(perseverative +

362 regressive errors $)$ compared to sham females $(0.11 \pm 0.13$ vs. $0.49 \pm 0.12, p<0.05$, unpaired t-test $)$.

363 (F) Sham females accumulated rewards after the first correct Reversal trial faster than pOVX

364 females (best fit line with 95\% C.I. plotted). (G) RL model applied to odor-based multiple choice

365 reversal task. Schematic based on (Verharen et al., 2019b). (H) Best-fit $\alpha$ learning rate estimates

366 did not significantly differ by task phase or treatment. (I) There was a significant interaction

367 between task phase and treatment group on the best-fit explore/exploit $\beta$ parameter (Two-way

368 ANOVA task phase $\times$ treatment interaction: $F(1,29)=7.101, \mathrm{p}<0.05)$. Post-hoc comparisons

369 revealed that $\beta$ parameter estimates were significantly higher during Reversal compared to the

370 Discrimination phase for sham ( $\mathrm{p}<0.0001$, uncorrected Fisher's LSD) and pOVX females $(\mathrm{p}<0.05$,

371 uncorrected Fisher's LSD). In addition, Reversal phase $\beta$ parameter estimates were significantly

372 lower in pOVX females compared to sham $(\mathrm{p}<0.05$, uncorrected Fisher's LSD). Data in $(\mathrm{H})$ plotted

373 as median $\pm \mathrm{IQR}$.

374 Prepubertal OVX is associated with increased intrinsic excitability of D2R(+) SPNs 
The DMS has been implicated in action selection and determining choice policy, and previous studies have found evidence that estrous cycle modulates the intrinsic excitability of striatal SPNs. Furthermore, several lines of evidence suggest that D2R(+) iSPNs: 1) are modulated by ovarian hormones (Le Saux and Di Paolo, 2005; Le Saux et al., 2006; Krentzel et al., 2019) and 2015; Delevich et al., 2020b). We therefore investigated whether changes in the intrinsic excitability of D2R(+) SPNs within DMS may contribute to sham vs. pOVX differences in choice policy during reversal learning. We performed whole-cell current clamp recordings of visually identified eGFP+ and neurons within the DMS of adult D2-eGFP transgenic female mice who underwent pOVX or sham surgery and unmanipulated female mice in the presence of the excitatory synaptic blockers NBQX and AP5 (Fig. 2A-C). AlexaFluor-594 was included in the internal solution, and all cells included in analysis were confirmed to have spinous morphology.

387 We found a main effect of treatment on D2-eGFP(+) SPN input resistance [main effect of treatment: $\mathrm{H}=8.76, \mathrm{p}=0.0125]$ with $\mathrm{pOVX}$ females exhibiting higher input resistance compared to sham and unmanipulated females (pOVX vs. sham $\mathrm{p}=0.013$; pOVX vs. unman. $\mathrm{p}=0.009$, uncorrected Dunn's test) (Fig. 2D). When we injected a series of positive current steps (Fig. 2E),

391 we found that the minimum amount of current necessary to trigger an action potential (rheobase)

392 was significantly lower in pOVX females compared to sham and unmanipulated females (pOVX 393 vs. sham $\mathrm{p}=0.001$; pOVX vs. unman. $\mathrm{p}=0.003$, uncorrected Fisher's LSD) (Fig. 2F). In addition, 394 there was a significant interaction between treatment and current on spike output $[\mathrm{F}(98,1715)=$ 2.517, p <0.0001] (Fig. 2G). While input-output curves were shifted leftward in pOVX compared to sham and unmanipulated females, there was no significant effect of treatment on maximum

397 firing rate $[\mathrm{F}(2,18.68)=0.10, \mathrm{p}=0.90]$ (Fig. $2 \mathrm{H})$. Finally, resting membrane potential $(\mathrm{RMP}) \mathrm{did}$ 
399 SPNs within the DMS are more intrinsically excitable in pOVX females compared to unstaged

400 sham and unmanipulated female mice.

A

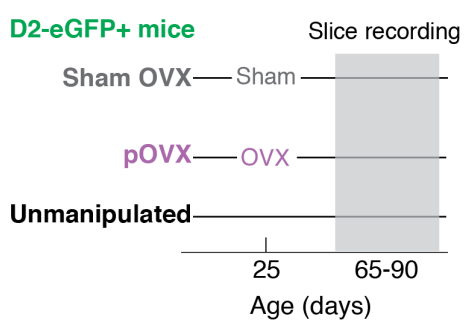

D

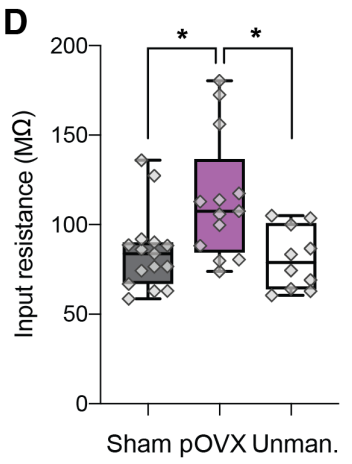

G

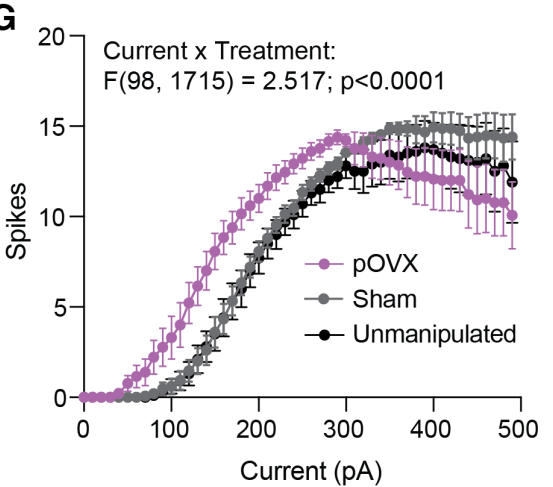

B

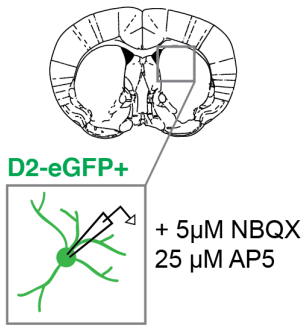

E
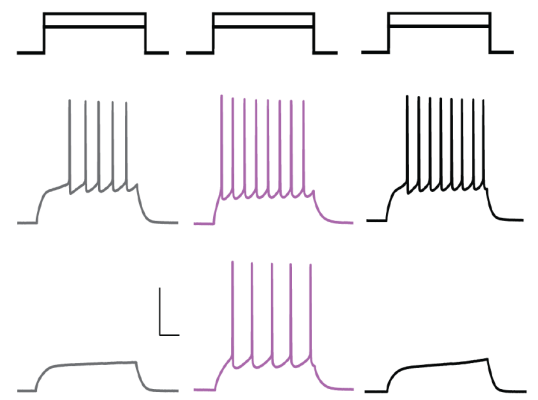

H

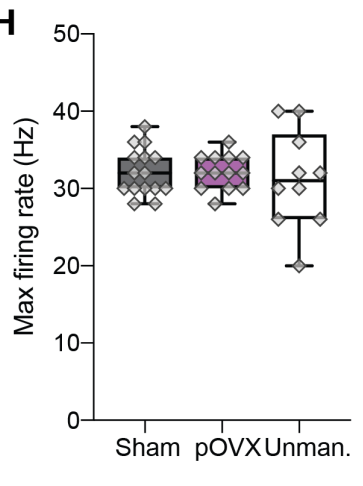

C

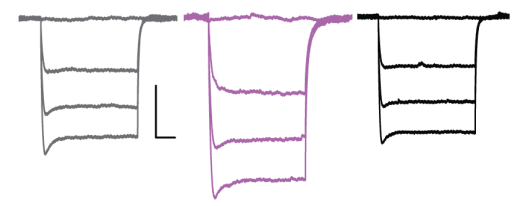

$\mathbf{F}$
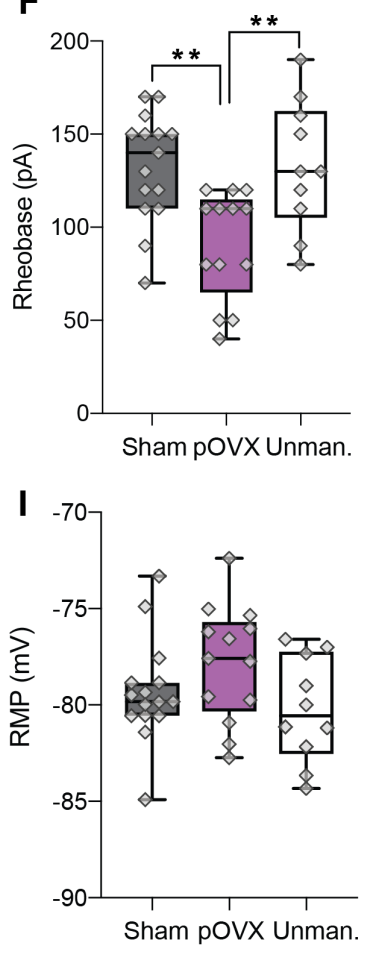

synaptic blockers. (A) At P25 D2-eGFP(+) female mice underwent sham or pOVX surgery, while recordings were made from visually identified D2-eGFP(+) SPNs within the DMS from all groups in adulthood (P65-90). (C) Representative responses to negative current steps (-150, -100, -50, 0 $\mathrm{pA}$ ) in D2R(+) SPNs from sham, pOVX, and unmanipulated females. Scale bar: $100 \mathrm{~ms}, 5 \mathrm{mV}$. 
407 (D) D2-eGFP(+) SPNs in pOVX female mice had higher input resistance compared to sham and 408 unmanipulated females. (E) Representative responses to positive current steps $(120,180 \mathrm{pA})$ in

409 D2R(+) SPNs from sham, pOVX, and unmanipulated females. Scale bar: $100 \mathrm{~ms}, 50 \mathrm{mV} .($ F)

410 Decreased rheobase of D2-eGFP(+) SPNs was observed in pOVX compared to sham and

411 unmanipulated females. (G) Spike number across sequential depolarizing current steps (10-500

$412 \mathrm{pA}$ ) for D2-eGFP(+) SPNs. Increased spiking was observed in pOVX compared to sham and

413 unmanipulated females (Two-way RM ANOVA current $\mathrm{x}$ treatment interaction: $\mathrm{F}(98,1715)=$

414 2.52, $\mathrm{p}<0.0001$ ). (H) No difference in maximum firing rate was observed across treatment groups.

415 (I) No difference in RMP was observed across treatment groups. ${ }^{*} \mathrm{p}<0.05,{ }^{*} \mathrm{p}<0.01 ; \mathrm{n} / \mathrm{N}=15 / 5$,

$41613 / 5$, and 10/3 for sham, pOVX, and unmanipulated mice, respectively.

417 Chemogenetic activation of D2R(+) SPNs in DMS reduces perseverative bias and promotes

Given that OVX females exhibit a more exploratory reversal strategy and greater intrinsic excitability of D2R(+) SPNs in DMS, we next asked whether experimentally increasing D2R(+)

421 SPN intrinsic excitability would similarly bias intact female mice towards increased exploration

422 during the Reversal phase. Female D2-Cre mice were bilaterally infused with $0.5 \mu \mathrm{L}$ of Cre423 dependent DREADD virus (hM4Di-mCherry or hM3Dq-mCherry) and trained 4-6 weeks later in 424 the 4 choice odor-based reversal learning task (Fig. 3A). Female mice expressing Cre-inducible 425 mCherry were used to control for any effects of surgery, AAV infection, and clozapine-N-oxide 426 (CNO) administration on behavior. To examine how CNO activation of hM3Dq expressed by $427 \mathrm{D} 2 \mathrm{R}(+)$ SPNs in DMS alters their activity, we performed whole-cell current clamp recordings of 428 identified mCherry+ neurons in mice that expressed the excitatory DREADD hM3Dq or mCherry 429 alone (Fig. 3B). Briefly, spike output in response to depolarizing steps (0-360 pA, 20 pA steps) 
430 was recorded from visually identified mCherry+ neurons in D2-mCherry or D2-hM3Dq-mCherry

431 expressing SPNs in DMS (Fig. 3A-D). Next, $10 \mu \mathrm{M}$ CNO was bath-applied for 5 minutes and

432 spike output to the same sequential series of depolarizing current steps was recorded (Fig. 3A-D).

433 There was no significant interaction between current step and drug on spike output in D2-mCherry

434 expressing SPNs [Two-way RM ANOVA, current $x$ drug: $F(18,36)=0.89, p=0.59$ ] (Fig. 3C) but

435 there was a significant interaction between current and drug on spike output in D2-hM3Dq-

436 mCherry SPNs [Two-way RM ANOVA, current x drug: $F(18,36)=3.93, p=0.0002]$ (Fig. 3D).

437 Finally, there was a significant interaction between virus and drug on rheobase [Two-way RM

438 ANOVA $F(1,4)=16.0, p=0.016]$ (Fig. 3E).
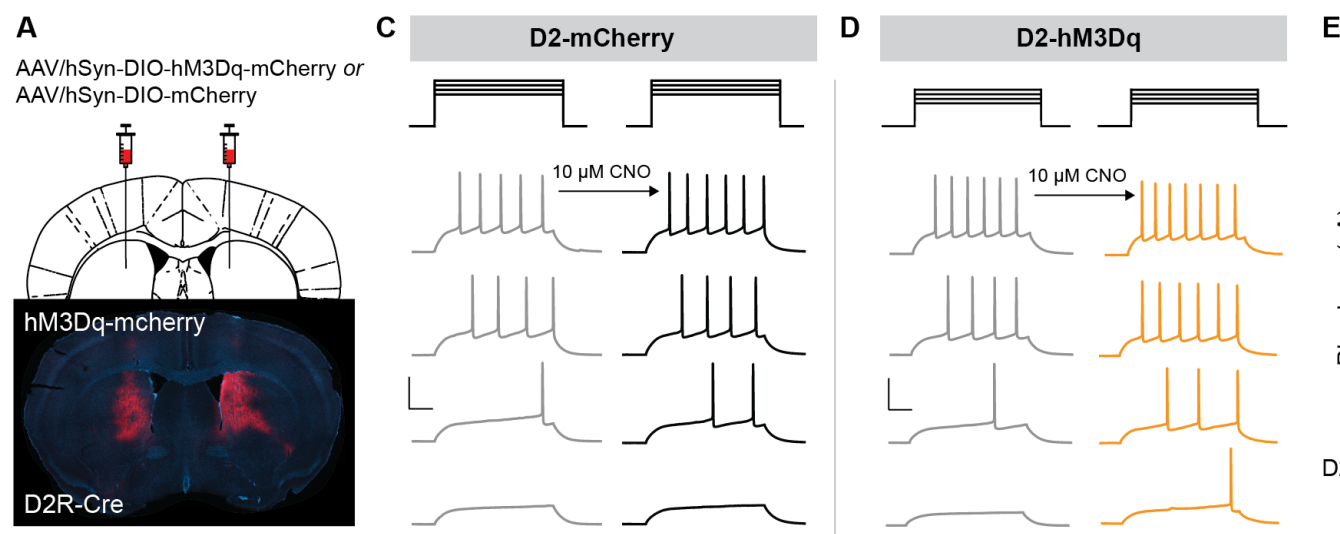

E Virus $x$ drug:
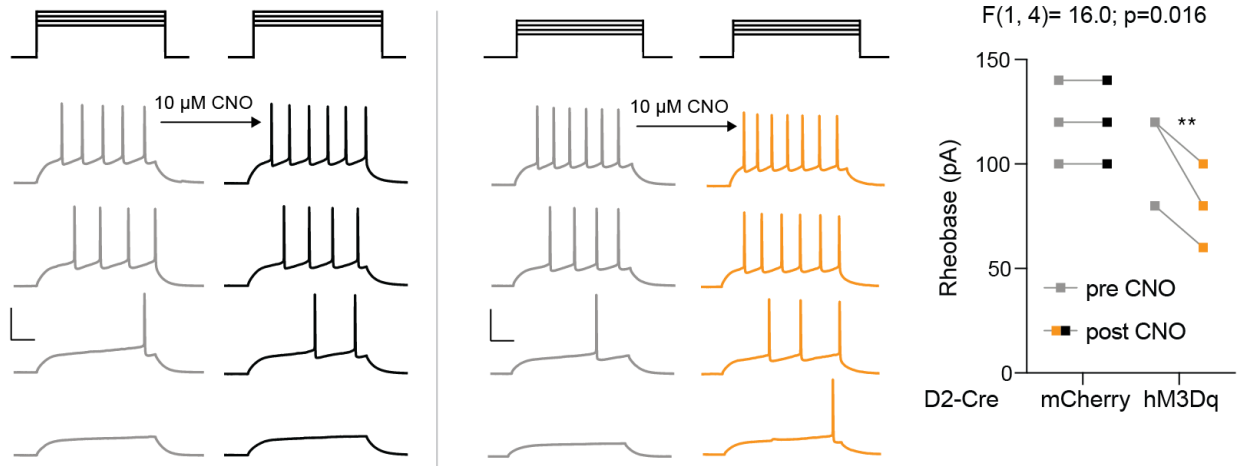

B
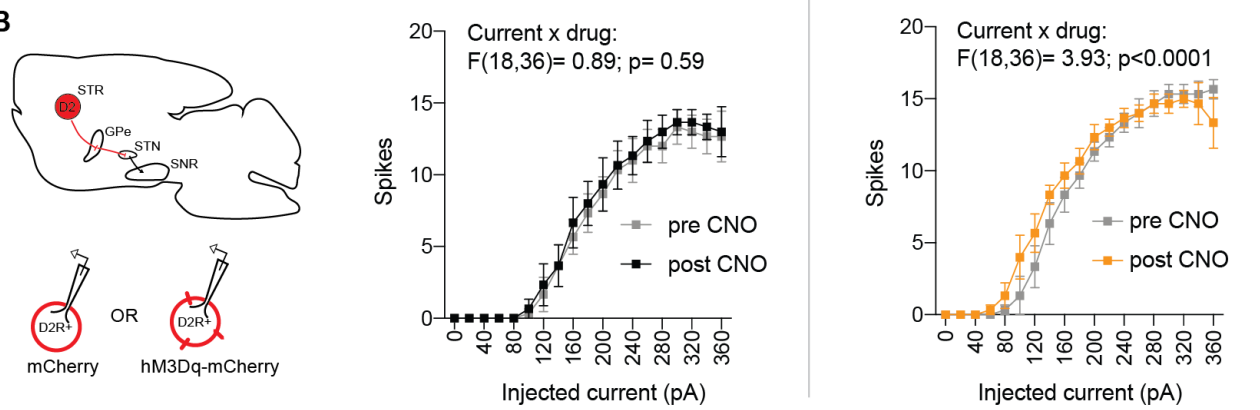

439 Figure 3. CNO increases intrinsic excitability of hM3Dq-expressing iSPNs. (A) Schematic of

440 injection and representative brain section showing hM3Dq-mCherry expression in the DMS of

441 D2-Cre mouse. (B) Schematic of indirect pathway expression (sagittal view) and whole-cell patch-

442 clamp configuration of mCherry+ or hM3Dq-mCherry+ iSPNs in female D2-Cre mice. (C) Top 
443 panel: representative responses to positive current steps $(100,120,140,160 \mathrm{pA})$ in mCherry+

444 iSPNs before and after CNO wash on. Scale bar: $100 \mathrm{~ms}, 50 \mathrm{mV}$. Bottom panel: no significant

445 interaction between current step and CNO treatment on spike output in $\mathrm{D} 2 \mathrm{R}(+)$ mCherry-

446 expressing iSPNs. (D) Top panel: representative responses to positive current steps (100, 120, 140,

$447160 \mathrm{pA}$ ) in hM3Dq-mCherry+ iSPNs before and after CNO wash on. Scale bar: $100 \mathrm{~ms}, 50 \mathrm{mV}$.

448 Bottom panel: significant interaction between current step and CNO treatment on spike output in

Summary of CNO wash on effect on rheobase (Two-way ANOVA virus $\times$ drug, $\mathrm{p}<0.05$ ).

454 (Odor 1) on 8/10 consecutive trials. Twenty-four hours later, all groups were administered CNO

455 (1.0 mg/kg, i.p.) and tested for their recall of discrimination learning followed immediately by a

456 Reversal phase in which Odor 1 was no longer rewarded and Odor 2 became rewarded. There was

457 a significant effect of task phase on trials to criterion [Reversal vs. Discrimination; $F(1,17)=16.58$,

$458 \mathrm{p}=0.0008]$ but no significant effect of virus $[\mathrm{F}(2,17)=0.37, \mathrm{p}=0.69]$ or interaction between virus

459 and task phase $[\mathrm{F}(2,17)=0.09, \mathrm{p}=0.92]$ (Fig. 4B). While there was no significant effect of

460 chemogenetic manipulation on Reversal phase trials to criterion, we found a significant interaction

461 between virus and error type during Reversal $[F(10,85)=2.721, p=0.006]($ Fig. $4 \mathrm{C})$ that was absent

462 during Discrimination when mice were on saline (Supplementary Figure 2). D2-hM3Dq mice

463 made significantly fewer perseverative errors compared to D2-mCherry ( $\mathrm{p}=0.015$, uncorrected

464 Dunn's test) and D2-hM4Di groups ( $\mathrm{p}=0.028$, uncorrected Fisher's LSD) and made significantly

465 more regressive errors compared to D2-hM4Di mice ( $\mathrm{p}=0.03$, uncorrected Fisher's LSD) (Fig. 
4C). We next examined whether chemogenetic manipulation of D2R(+) neurons in the DMS altered Reversal error bias within mice. There was a significant effect of virus on Reversal error bias $(\mathrm{H}=9.06, \mathrm{p}=0.005$ Kruskal-Wallis test $)$, with D2-hM3Dq mice having a significantly lower Reversal error bias compared to D2-mCherry ( $\mathrm{p}=0.019$, uncorrected Dunn's test) and D2-hM4Di groups ( $\mathrm{p}=0.005$, uncorrected Dunn's test) (Fig. 4D), consistent with a greater tendency to make

471 regressive errors compared to perseverative errors. This data suggests that chemogenetic activation 472 of $\mathrm{D} 2 \mathrm{R}(+)$ neurons in the DMS produced a pattern of reversal phase choice behavior that was 473 similar to the effect seen in pOVX mice. parameters might explain the pattern of reversal behavior we observed in pOVX female mice and D2-hM3Dq female mice. Fitting the same RL model (task phase-specific $\alpha$ and $\beta$ parameters; see Methods) we found that there was no significant interaction between virus and task phase on learning rate $\alpha[F(2,17)=0.45, \mathrm{p}=0.64]$ (Fig. $4 \mathrm{E})$, but there was a significant interaction between

479 virus and task phase for the explore/exploit parameter $\beta[F(2,17)=5.18, p=0.018]$ (Fig. $4 F)$. The 480 Reversal phase explore/exploit parameter was significantly lower in D2-hM3Dq mice compared 481 to D2-mCherry ( $\mathrm{p}=0.0002$, uncorrected Fisher's LSD) and D2-hM4Di $(\mathrm{p}=0.002$, uncorrected 482 Fisher's LSD) (Fig. 4F). These data suggest that chemogenetic activation of D2R $(+)$ neurons 483 within DMS biases choice strategy in female mice to be more exploratory during reversal learning. 484 Moreover, chemogenetic activation of $\mathrm{D} 2 \mathrm{R}(+)$ neurons within the DMS produced behavior in 485 female mice that mimicked the behavioral pattern seen in OVX females, including a reduction in 486 Reversal error bias during reversal learning and a reduced explore/exploit $\beta$ parameter consistent 487 with a less exploitative, more exploratory choice policy. Taken together with evidence that D2R(+) 488 iSPNs within the DMS are more intrinsically excitable in pOVX compared to sham females, these 

modulating iSPN intrinsic excitability within DMS (Fig. 5).

A

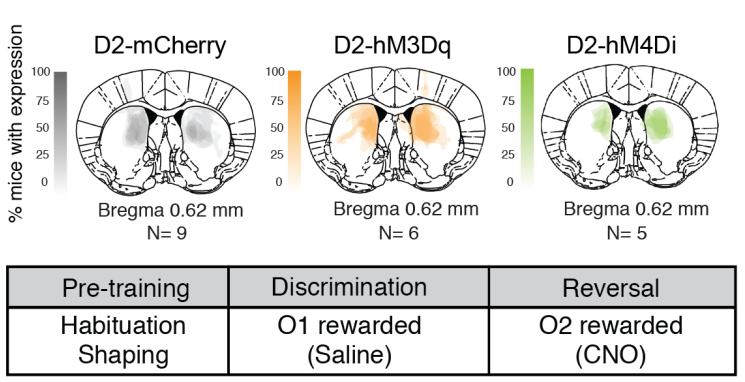

C

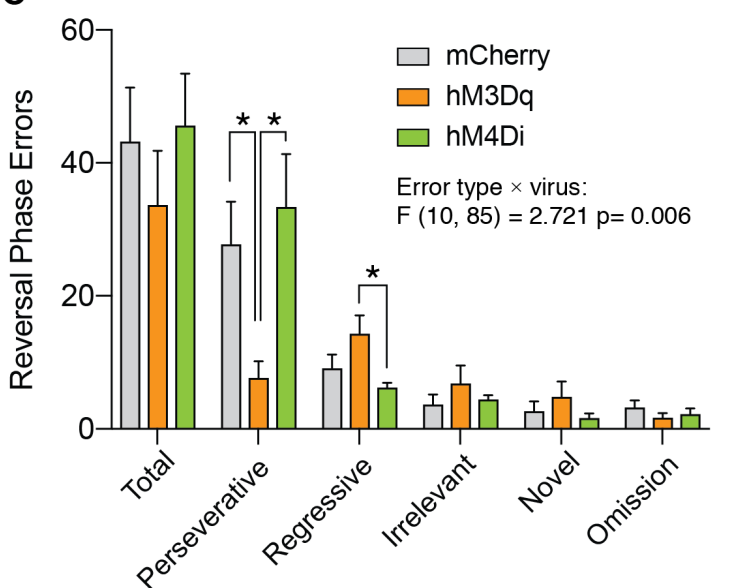

B

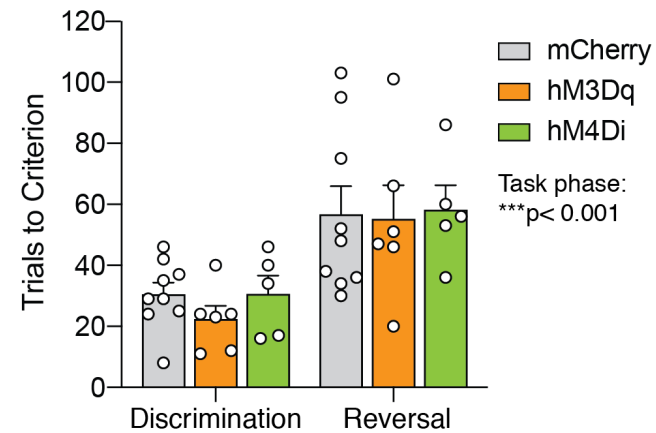

D
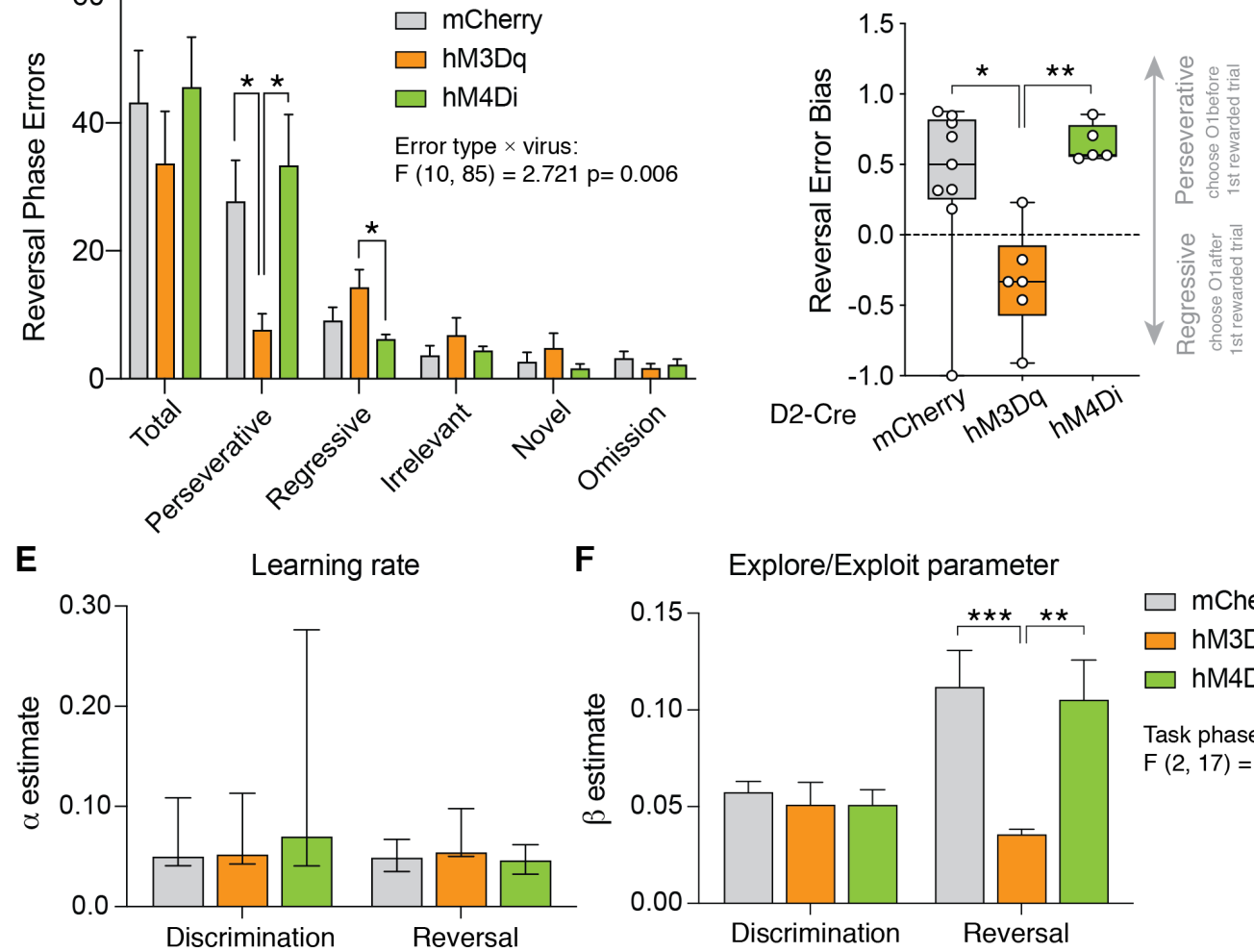

F Explore/Exploit parameter

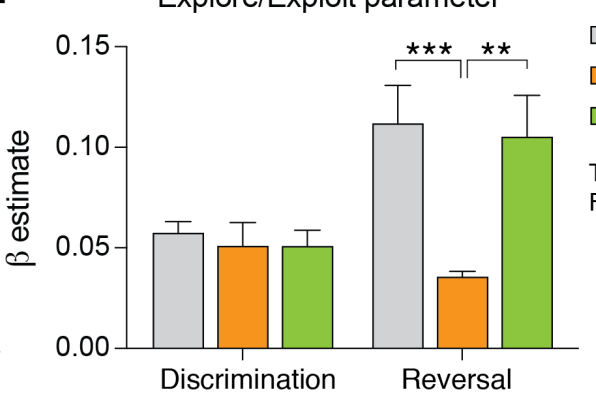

$\square$ mCherry

$\square \mathrm{hM} 3 \mathrm{Dq}$

$\square \mathrm{hM} 4 \mathrm{Di}$

Task phase $\times$ virus:

$F(2,17)=5.18 ; p=0.018$

Figure 4. Chemogenetic activation of $\mathrm{D} 2 \mathrm{R}(+)$ neurons in DMS promotes a more exploratory

492 reversal strategy in intact female mice. (A) Top panel: schematic illustrating injection site and

493 viral spread female D2-Cre DIO-mCherry (N=9), DIO-hM3Dq (N=6), and DIO-hM4Di (N=5)

494 mice. Bottom panel: summary of behavioral training. (B) There was a main effect of task phase

495 but no effect of virus on trials to criterion (Two-way RM ANOVA main effect of task phase: 
$\mathrm{F}(2,17)=16.58, \mathrm{p}<0.001)$. (C) There was a significant interaction between error type and virus on reversal errors (Two-way RM ANOVA error type $\times$ manipulation interaction: $F(10,85)=2.72$,

$498 \mathrm{p}<0.01)$ with D2-hM3Dq mice making fewer perseverative errors $(\mathrm{p}<0.05$, uncorrected Fisher's

499 LSD) compared to D2-mCherry and D2-hM4di mice and more regressive errors compared to D2-

500 hM4di mice ( $<<0.05$, uncorrected Fisher's LSD). (D) There was a main effect of virus on Reversal

501 error bias ( $\mathrm{W}=9.06, \mathrm{p}<0.01$ Kruskal-Wallis test), with $\mathrm{D} 2-\mathrm{hM} 3 \mathrm{Dq}$ mice showing reduced bias for

502 perseverative errors compared to D2-mCherry ( $\mathrm{p}<0.05$, uncorrected Dunn's test) and D2-hM4Di

503 mice $(\mathrm{p}<0.01$, uncorrected Dunn's test). (E) Best-fit $\alpha$ learning rate did not significantly differ by

504 task phase or virus. (F) There was a significant interaction between task phase and treatment group

505 on the best-fit explore/exploit parameter $\beta$ (Two-way ANOVA task phase $\mathrm{x}$ treatment interaction:

$506 \mathrm{~F}(2,17)=5.18, \mathrm{p}<0.05)$. Post-hoc comparisons revealed that $\beta$ parameter estimates were

507 significantly higher during Reversal compared to Discrimination phase for D2-mCherry mice

$508(\mathrm{p}<0.01$, uncorrected Fisher's LSD) and D2-hM4Di mice $(\mathrm{p}<0.05$, uncorrected Fisher's LSD) but

509 not D2-hM3Dq mice ( $\mathrm{p}=0.41$, uncorrected Fisher's LSD). In addition, Reversal phase $\beta$ parameter

510 estimates were significantly lower in D2-hM3Dq mice compared to D2-mCherry $(\mathrm{p}<0.001$,

511 uncorrected Fisher's LSD) and D2-hM4Di mice ( $<<0.01$, uncorrected Fisher's LSD). ${ }^{*} p<0.05$,

$512 * * \mathrm{p}<0.01, * * * \mathrm{p}<0.001$. Data in (E) plotted as median $\pm \mathrm{IQR}$. 


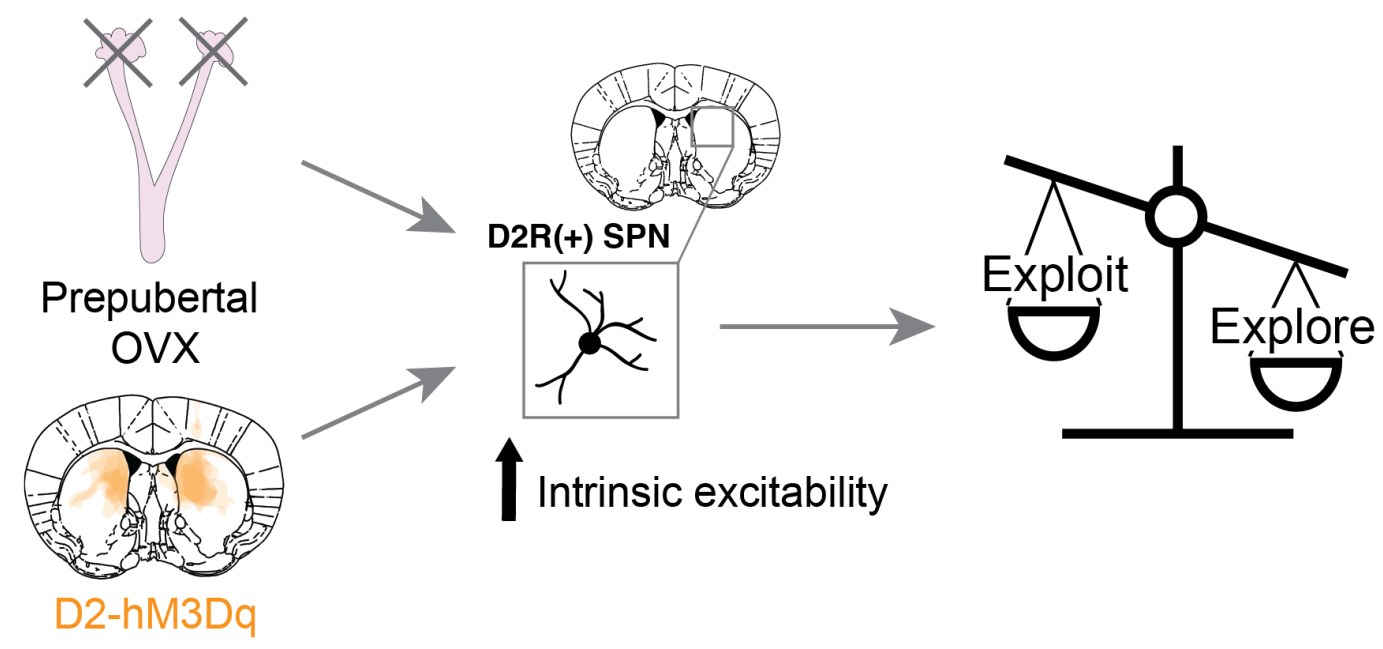

516 Figure 5. Results summary. Both pOVX and chemogenetic activation of D2R(+) neurons within

517 the DMS are associated with increased intrinsic excitability of iSPNs. In turn, both manipulations

518 are associated with less perseverative, more exploratory choice strategy during reversal learning.

519 While indirect, the convergent behavioral effects of pOVX and chemogenetic activation of DMS

$520 \mathrm{D} 2 \mathrm{R}(+)$ neurons suggest that the increased intrinsic excitability of iSPNs in the DMS of pOVX

521 mice could contribute to the altered reversal strategy observed. Future experiments should perform

522 in vivo recording of $\mathrm{D} 2 \mathrm{R}(+)$ SPNs and/or chemogenetic manipulation experiments in pOVX

523 females to probe the relationship between altered D2R $(+)$ SPN intrinsic properties and reversal

524 learning strategy on a trial-by-trial basis.

525

526

527

528

529

530

531

532

533

\section{Discussion:}

We found that pOVX altered how female mice solved a reversal learning task. Using RL models fit to trial-by-trial behavioral data, we found that pOVX mice exhibited a more exploratory choice policy during reversal learning than sham controls, captured by a lower inverse temperature $\beta$ parameter. This difference in exploratory choice behavior was accompanied by increased intrinsic excitability of $\mathrm{D} 2 \mathrm{R}(+)$ iSPNs in the DMS, a region that is implicated in regulating action selection and choice policy. We then sought to mimic this effect using chemogenetics. We 
534 demonstrated that chemogenetic activation of D2R(+) neurons in vitro similarly enhanced iSPN

535 intrinsic excitability in slices from female brains. In addition, activation of DMS D2R(+) neurons

536 in vivo decreased the ratio of perseverative to regressive errors and promoted exploratory choice

537 captured by a lower inverse temperature $\beta$ parameter. Together, these data suggest that two distinct

manipulations: pOVX and hM3Dq activation converged on similar behavioral effects through a

shared mechanism of enhancing DMS iSPN intrinsic excitability.

Our data are consistent with studies that manipulate D2Rs and model choice policy.

541 Germline D2R knockout (Kwak et al., 2014), systemic D2R antagonist administration (Eisenegger

542 et al., 2014), and intrastriatal D2R antagonist infusion (Lee et al., 2015) are each associated with

543 more exploratory choice policy. However, none of these studies could rule out the contribution of

544 presynaptic D2 autoreceptors, which is important given the apparent role of tonic dopamine in

545 modulating explore/exploit balance (Beeler et al., 2010; Humphries et al., 2012; Cinotti et al.,

546 2019), but see (Costa et al., 2014). Our chemogenetic manipulation experiments (which do not

547 infect D2R(+) dopamine axon terminals) clearly demonstrate that activation of D2R(+) neurons

548 within DMS is sufficient to bias performance towards exploration.

We speculate that there are two likely circuit mechanisms downstream of D2R(+) iSPNs

550 that may be responsible for promoting exploratory choice policy. The first involves local lateral

551 connections from iSPNs to direct pathway SPNs (dSPNs) and the second involved the interface of

552 the direct and indirect pathways in basal ganglia output centers such as the substantia nigra pars

553 reticulata $(\mathrm{SNr})$. One recent study showed that systemic injection of the $\mathrm{D} 2 \mathrm{R}$ antagonist raclopride

554 induced dopamine-dependent transcriptional activation in iSPNs that opposed the activation of

555 dSPNs, suggesting that iSPN to dSPN transmodulation is an important mechanism for behavioral

556 flexibility (Matamales et al., 2020). Therefore, it is possible that elevated iSPN activity, either 
557 through pOVX or chemogenetic activation, promotes exploratory choice by dampening the 558 activity of task-relevant ensembles of dSPNs that would normally promote the selection of the 559 highest estimated-value option. Opponent mechanisms between the direct and indirect pathway at 560 convergent downstream targets are also predicted to regulate choice policy (Collins and Frank, $5612014)$.

Studies have shown that the intrinsic properties of striatal SPNs differ between females and males before puberty (Dorris et al., 2015) and in an estrous cycle-dependent manner after puberty

564 (Proano et al., 2018). Interestingly, Proano et al. found that the intrinsic excitability of accumbal 565 SPNs was significantly higher during diestrus/metestrus, when estradiol and progesterone levels are low, compared to proestrus/estrus (Proano et al., 2018). This may be in keeping with our

567 observation that the intrinsic excitability of D2R(+) SPNs was higher in pOVX females compared 568 to sham females, since pOVX females lack gonadally-produced estradiol and progesterone.

569 Estradiol has also been shown to influence dopamine release and reuptake in the striatum (Calipari 570 et al., 2017), including the dorsal striatum (Becker and Beer, 1986; Becker, 1990). Recent data

571 also suggest that dopamine influences the postnatal maturation of intrinsic excitability of SPN 572 populations within the striatum (Lieberman et al., 2018). Therefore, it is possible that the changes 573 we observed in D2R(+) SPN excitability in pOVX female mice may occur by direct action on 574 SPNs or downstream of hormonal effects on presynaptic dopamine release (Lin et al., 2020). 578 OVX (Le Saux et al., 2006). The aforementioned treatments did not alter D2R mRNA expression, 579 suggesting that estradiol modulates D2R binding through a mechanism other than transcriptional 
580 regulation of D2R expression. Furthermore, OVX reduces the expression of preproenkephalin,

581 which produces the endogenous opioid peptide, enkephalin, which is expressed in iSPNs (Le Saux

582 and Di Paolo, 2005). Again, the effect of OVX on preproenkephalin expression can be

583 counteracted by estradiol administration. Finally, in the nucleus accumbens core, rapid

584 enhancement of mEPSC amplitude by estradiol is inversely correlated with rheobase (Krentzel et

585 al., 2019). Given that D2R(+) SPNs typically display lower rheobase compared to D1R+ SPNs,

586 this suggests that estradiol may exert a greater effect on D2R(+) iSPNs compared to D1R(+)

587 dSPNs. However, it should be noted that the authors did not observe rapid effects of estradiol on

588 mEPSC amplitude within dorsal striatum (Krentzel et al., 2019).

There are several limitations to our current study that should be noted. First, we cannot

590 assume that the changes we observed in SPN intrinsic excitability are specific to the dorsomedial

591 region of the striatum or to the D2R(+) SPN cell type. Second, our evidence linking the increased

592 excitability of $\mathrm{D} 2 \mathrm{R}(+)$ SPNs in DMS to the more exploratory choice strategy used by pOVX

593 females is correlational. In independent experiments we observed that 1) pOVX promoted

594 exploratory choice strategy during Reversal 2) pOVX is associated with elevated intrinsic

595 excitability of D2R(+) SPNs in DMS and 3) that chemogenetic activation of D2R(+) SPNs in DMS

596 promoted exploratory choice strategy during Reversal. In the future, more direct evidence could

597 be gained by performing manipulation experiments to reduce the activity of DMS D2R(+) SPNs

598 in OVX females, or by recording the activity of these same neurons in pOVX and sham females

599 during behavior. Finally, while we performed OVX prior to puberty onset, we do not know whether

600 the timing of OVX plays an important role in the observed effect on behavior and physiology. We

601 also do not know if and when hormone replacement may rescue the effects of pOVX. Future

602 studies should examine timing effects of OVX and hormone replacement on these outcome 
measures. Still, in light of these limitations, our data suggest future lines of inquiry into the

604 relationship between puberty, ovarian hormones, SPN physiology, and choice policy in value-

605 based decision making.

As yet, we have not identified the mechanism by which pOVX leads to enhanced

610 in Kir2 family inward rectifying K+ channels (Uchimura et al., 1989; Nisenbaum and Wilson,

611 1995; Mermelstein et al., 1998; Shen et al., 2007), and a reduction in dendritic length/complexity

612 has been associated with reduced Kir2 expression and increased intrinsic excitability (Cazorla et

613 al., 2012; Sebel et al., 2017). Therefore, it would be informative to compare iSPN Kir2 channel

614 currents and dendritic morphology in sham vs. pOVX females. Finally, it is possible that the

615 increase in intrinsic excitability of the $\mathrm{D} 2 \mathrm{R}(+)$ SPNs in pOVX females could represent a 616 homeostatic plasticity mechanism that accompanies a reduction in excitatory synaptic inputs to 617 them, but we did not measure synaptic inputs onto D2R(+) SPNs in this study. 
626 biased choice strategies utilized during value-based decision making. While previous studies have

627 separately provided evidence that ovarian hormones regulate the intrinsic excitability of SPNs and

628 aspects of value-based decision making, we show for the first time that pOVX alters

629 explore/exploit balance of choice strategy while also increasing the intrinsic excitability of D2R $(+)$

630 SPNs in the DMS. These data suggest that pubertal status may influence explore/exploit balance

631 via the modulation of SPN intrinsic excitability within the DMS and highlight a role for ovarian

632 hormones in establishing sex-specific decision-making strategies in adulthood. These data can

633 inform the basic science of decision making and the study of the many psychiatric disorders that

634 emerge after puberty and also show sex differences in their prevalence or manifestation.

\section{Acknowledgments}

636 We thank Yuting Zhang and Kenechukwu Okwuosa for technical assistance with mouse behavior

637 testing. We thank Benjamin Hoshal for contributing to analysis code. We thank Dr. Helen Bateup

638 for feedback on the manuscript and Dr. Anne Collins and Wilbrecht lab members for helpful

639 discussion.

640 Declaration of interests

641 The authors declare that there are not conflicts of interest. 


\section{References:}

Addicott MA, Pearson JM, Sweitzer MM, Barack DL, Platt ML (2017) A Primer on Foraging and the Explore/Exploit Trade-Off for Psychiatry Research. Neuropsychopharmacol 42:1931-1939.

Almey A, Filardo EJ, Milner TA, Brake WG (2012) Estrogen Receptors Are Found in Glia and at Extranuclear Neuronal Sites in the Dorsal Striatum of Female Rats: Evidence for Cholinergic But Not Dopaminergic Colocalization. Endocrinology 153:5373-5383.

Alonso-Caraballo Y, Ferrario CR (2019) Effects of the estrous cycle and ovarian hormones on cue-triggered motivation and intrinsic excitability of medium spiny neurons in the Nucleus Accumbens core of female rats. Horm Behav 116.

Becker JB (1990) Direct Effect of 17-Beta-Estradiol on Striatum - Sex-Differences in Dopamine Release. Synapse 5:157-164.

Becker JB, Beer ME (1986) The Influence of Estrogen on Nigrostriatal Dopamine Activity Behavioral and Neurochemical Evidence for Both Pre-Naptic and Postsynaptic Components. Behav Brain Res 19:27-33.

Beeler JA, Daw N, Frazier CRM, Zhuang XX (2010) Tonic dopamine modulates exploitation of reward learning. Front Behav Neurosci 4.

Calipari ES, Juarez B, Morel C, Walker DM, Cahill ME, Ribeiro E, Roman-Ortiz C, Ramakrishnan C, Deisseroth K, Han MH, Nestler EJ (2017) Dopaminergic dynamics underlying sex-specific cocaine reward. Nat Commun 8.

Cazorla M, Shegda M, Ramesh B, Harrison NL, Kellendonk C (2012) Striatal D2 Receptors Regulate Dendritic Morphology of Medium Spiny Neurons via Kir2 Channels. J Neurosci 32:2398-2409.

Chen CS, Ebitz RB, Bindas SR, Redish AD, Hayden BY, Grissom NM (2021) Divergent Strategies for Learning in Males and Females. Curr Biol 31:39-+.

Chen JR, Yan YT, Wang TJ, Chen LJ, Wang YJ, Tseng GF (2009) Gonadal Hormones Modulate the Dendritic Spine Densities of Primary Cortical Pyramidal Neurons in Adult Female Rat. Cereb Cortex 19:2719-2727.

Cinotti F, Fresno V, Aklil N, Coutureau E, Girard B, Marchand AR, Khamassi M (2019) Dopamine blockade impairs the exploration-exploitation trade-off in rats. Sci Rep-Uk 9.

Cohen JD, McClure SM, Yu AJ (2007) Should I stay or should I go? How the human brain manages the trade-off between exploitation and exploration. Philos T R Soc B 362:933942.

Collins AG, Frank MJ (2014) Opponent actor learning (OpAL): modeling interactive effects of striatal dopamine on reinforcement learning and choice incentive. Psychological review 121:337-366.

Costa VD, Tran VL, Turchi J, Averbeck BB (2014) Dopamine Modulates Novelty Seeking Behavior During Decision Making. Behav Neurosci 128:556-566.

Daw ND, O'Doherty JP, Dayan P, Seymour B, Dolan RJ (2006) Cortical substrates for exploratory decisions in humans. Nature 441:876-879.

Delevich K, Hall CD, Piekarski D, Zhang YT, Wilbrecht L (2020a) Prepubertal gonadectomy reveals sex differences in approach-avoidance behavior in adult mice. Horm Behav 118.

Delevich K, Hoshal BD, Zhang Y, Vedula S, Collins AGE, Wilbrecht L (2020b) Activation But Not Inhibition of the Indirect Pathway Disrupts Choice Suppression in a Freely Moving, Multiple Choice Foraging Task. In: Available at SSRN. 
Dorris DM, Cao JY, Willett JA, Hauser CA, Meitzen J (2015) Intrinsic excitability varies by sex in prepubertal striatal medium spiny neurons. J Neurophysiol 113:720-729.

Dunovan K, Verstynen T (2016) Believer-Skeptic Meets Actor-Critic: Rethinking the Role of Basal Ganglia Pathways during Decision-Making and Reinforcement Learning. Front Neurosci-Switz 10.

Eckstein MK, Master SL, Dahl RE, Wilbrecht L, Collins AGE (2021) The Unique Advantage of Adolescents in Probabilistic Reversal: Reinforcement Learning and Bayesian Inference Provide Adequate and Complementary Models. bioRxiv:2020.2007.2004.187971.

Eisenegger C, Naef M, Linssen A, Clark L, Gandamaneni PK, Muller U, Robbins TW (2014) Role of Dopamine D2 Receptors in Human Reinforcement Learning. Neuropsychopharmacol 39:2366-2375.

Frank MJ, Doll BB, Oas-Terpstra J, Moreno F (2009) Prefrontal and striatal dopaminergic genes predict individual differences in exploration and exploitation. Nat Neurosci 12:1062U1145.

Gopnik A (2020) Childhood as a solution to explore-exploit tensions. Philos T R Soc B 375.

Gould E, Woolley CS, Frankfurt M, Mcewen BS (1990) Gonadal-Steroids Regulate Dendritic Spine Density in Hippocampal Pyramidal Cells in Adulthood. J Neurosci 10:1286-1291.

Humphreys KL, Lee SS, Telzer EH, Gabard-Durnam LJ, Goff B, Flannery J, Tottenham N (2015) Exploration-Exploitation Strategy is Dependent on Early Experience. Dev Psychobiol 57:313-321.

Humphries MD, Khamassi M, Gurney K (2012) Dopaminergic control of the explorationexploitation trade-off via the basal ganglia. Front Neurosci-Switz 6.

Johnson C, Wilbrecht L (2011) Juvenile mice show greater flexibility in multiple choice reversal learning than adults. Dev Cogn Neurosci 1:540-551.

Johnson CM, Peckler H, Tai LH, Wilbrecht L (2016) Rule learning enhances structural plasticity of long-range axons in frontal cortex. Nat Commun 7:10785.

Krentzel AA, Barrett LR, Meitzen J (2019) Estradiol rapidly modulates excitatory synapse properties in a sex- and region-specific manner in rat nucleus accumbens core and caudate-putamen. J Neurophysiol 122:1213-1225.

Krentzel AA, Willett JA, Johnson AG, Meitzen J (2021) Estrogen receptor alpha, G-protein coupled estrogen receptor 1, and aromatase: Developmental, sex, and region-specific differences across the rat caudate-putamen, nucleus accumbens core and shell. J Comp Neurol 529:786-801.

Kwak S, Huh N, Seo JS, Lee JE, Han PL, Jung MW (2014) Role of dopamine D2 receptors in optimizing choice strategy in a dynamic and uncertain environment. Front Behav Neurosci 8.

Le Saux M, Di Paolo T (2005) Chronic estrogenic drug treatment increases preproenkephalin mRNA levels in the rat striatum and nucleus accumbens. Psychoneuroendocrino 30:251260.

Le Saux M, Morissette M, Di Paolo T (2006) ER beta mediates the estradiol increase of D-2 receptors in rat striatum and nucleus accumbens. Neuropharmacology 50:451-457.

Lee E, Seo M, Dal Monte O, Averbeck BB (2015) Injection of a Dopamine Type 2 Receptor Antagonist into the Dorsal Striatum Disrupts Choices Driven by Previous Outcomes, But Not Perceptual Inference. J Neurosci 35:6298-6306.

Lenow JK, Constantino SM, Daw ND, Phelps EA (2017) Chronic and Acute Stress Promote Overexploitation in Serial Decision Making. J Neurosci 37:5681-5689. 
Lieberman OJ, McGuirt AF, Mosharov EV, Pigulevskiy I, Hobson BD, Choi S, Frier MD, Spiny Projection Neuron Excitability during a Critical Period. Neuron 99:540-+. 
Tansey EM, Arbuthnott GW, Fink G, Whale D (1983) Oestradiol-17-Beta Increases the Firing Rate of Antidromically Identified Neurons of the Rat Neostriatum. Neuroendocrinology 37:106-110.

Uban KA, Rummel J, Floresco SB, Galea LAM (2012) Estradiol Modulates Effort-Based Decision Making in Female Rats. Neuropsychopharmacol 37:390-401. Neurons. J Neurophysiol 62:1280-1286.

Verharen JPH, Adan RAH, Vanderschuren LJMJ (2019a) Differential contributions of striatal dopamine D1 and D2 receptors to component processes of value-based decision making. Neuropsychopharmacol 44:2195-2204.

Verharen JPH, Kentrop J, Vanderschuren LJMJ, Adan RAH (2019b) Reinforcement learning across the rat estrous cycle. Psychoneuroendocrino 100:27-31.

Wallace M, Luine V, Arellanos A, Frankfurt M (2006) Ovariectomized rats show decreased recognition memory and spine density in the hippocampus and prefrontal cortex. Brain Res 1126:176-182.

811 Xia L, Master S, Eckstein MK, Wilbrecht L, Collins A (2020) Learning under uncertainty

Watanabe S (2010) Asymptotic Equivalence of Bayes Cross Validation and Widely Applicable Information Criterion in Singular Learning Theory. J Mach Learn Res 11:3571-3594.

Woolley CS, Gould E, Frankfurt M, Mcewen BS (1990) Naturally-Occurring Fluctuation in 